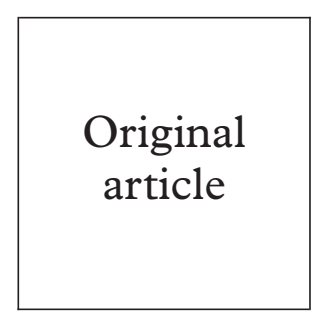

\title{
An investigation into the pathogenesis of vulvo-vaginal candidosis
}

\author{
S S El-Din, M T Reynolds, H R Ashbee, R C Barton, E G V Evans
}

Objective: To monitor yeasts isolated from women during and between episodes of recurrent vulvo-vaginal candidosis (VVC) to determine whether vaginal relapse or re-infection occurred. Methods: Women presenting at the genitourinary medicine clinic with signs and symptoms of VVC were recruited to the study $(n=121)$. A vaginal washing, high vaginal swab (HVS) and rectal swab were taken and the women treated with a single $500 \mathrm{mg}$ clotrimazole pessary. Women were asked to re-attend after 1,4 , and 12 weeks, or when the VVC recurred, when vaginal washings and HVS were repeated. Candida isolates recovered were strain typed using the $\mathrm{Ca} 3$ probe and their similarity assessed. Antifungal susceptibility to fluconazole and clotrimazole were determined.

Results: Of the women recruited, 47 completed the study, either returning for four visits or suffering a recurrence during the study period. Of the 22 women who experienced recurrence, the same strain was responsible for the initial and recurrent episode in 17 women. For the remaining five women, four had strain replacement and one had a change of species. None of the isolates recovered from the women demonstrated resistance to either clotrimazole or fluconazole.

Conclusions: Our findings support the theory of vaginal relapse and thus may support the use of more prolonged courses of antifungal therapy initially to increase the chances of eradication of the yeast.

(Sex Transm Inf 2001;77:179-183)

Keywords: vaginal candidosis

\section{Introduction}

Vulvo-vaginal candidosis (VVC) is a very common condition and most women will experience at least one episode during their reproductive years. ${ }^{1}$ A minority of women, however, suffer recurrent episodes of candidosis which can significantly affect their quality of life and their sexual health. While a number of factors are probably important in the pathogenesis of recurrent thrush, there are two main theories as to why some women experience frequent recurrences ${ }^{2}$ :

- Re-infection either from a sexual partner or a reservoir of yeasts in the gut

- Vaginal relapse due to incomplete eradication of the yeasts.

Several groups have found that in most women with vaginal thrush, identical species can also be isolated from the rectum..$^{3-5}$ However, attempts to eradicate the carriage of yeasts in the gut have not been shown in controlled trials to reduce significantly the number of symptomatic vaginal recurrences. ${ }^{6-9}$ Similarly, randomised controlled trials have shown that the treatment of male sexual partners with antifungals has no effect on the clearance and/or recurrence rate of vaginal candida in women. ${ }^{10-12}$ Some studies have used DNA fingerprinting methods to examine the genetic variability of yeasts isolated from women at successive symptomatic episodes of candida vaginitis, but in most of them samples were not taken between recurrences. ${ }^{13} 14$ Therefore, it was unclear whether the yeasts were eradicated after the initial therapy or merely persisted without causing symptoms.
The aim of this investigation was therefore to monitor the yeasts isolated from women not only during but also in between recurrent episodes of VVC, using molecular strain typing, to determine whether vaginal relapse or reinfection occurred. The possible role of antifungal resistance in VVC was also investigated.

\section{Methods}

STUDY POPULATION

A cohort of 121 premenopausal women attending the department of genitourinary medicine at Leeds General Infirmary with signs and symptoms of acute VVC were recruited into the study. Women who were less than 16 years of age, diabetic, HIV positive, or pregnant were excluded. Informed consent was obtained from the patient before entry. All patients underwent a full screen for sexually transmitted infections, including syphilis, gonorrhoea, chlamydia, Trichomonas vaginalis, and bacterial vaginosis and women with any of these infections were excluded. The initial diagnosis of VVC was made on clinical grounds. The examining doctor completed a questionnaire detailing age, history of similar episodes, medical history, contraceptives used, any medication taken, and symptoms and signs at presentation. A conventional high vaginal swab (HVS) and a rectal swab were taken from each woman, together with a $10 \mathrm{ml}$ vaginal washing using sterile water. The women were treated with a single $500 \mathrm{mg}$ clotrimazole pessary and asked to re-attend 1,4 , and 12 weeks after treatment, or if their symptoms recurred. The women were asked about symptoms, signs were recorded, and HVS and vaginal washings were repeated at each visit.
Accepted for publication 11 January 2001 
Table 1 Relation between symptoms and culture results in 121 women at their initial visit

\begin{tabular}{lllll}
\hline Variable & $\begin{array}{l}\text { Total } n=121 \\
(\%)\end{array}$ & $\begin{array}{l}\text { Culture positive } \\
n=78(\%)\end{array}$ & $\begin{array}{l}\text { Culture negative } \\
n=43(\%)\end{array}$ & $p$ Value \\
\hline $\begin{array}{l}\text { History } \\
\text { OCP }\end{array}$ & $53(43.8)$ & $30(56.6)$ & $23(43.4)$ & $0.16(\mathrm{NS})$ \\
$\quad$ HSE & $86(71.1)$ & $58(67.4)$ & $35(40.7)$ & \\
$\begin{array}{l}\text { Symptoms } \\
\text { Itching }\end{array}$ & $97(80.2)$ & $68(70.1)$ & $29(29.9)$ & 0.009 \\
$\quad \begin{array}{l}\text { Soreness } \\
\text { Discharge }\end{array}$ & $78(64.5)$ & $56(71.8)$ & $22(28.2)$ & 0.023 \\
$\quad \begin{array}{l}\text { Dysuria } \\
\text { Dyspareunia }\end{array}$ & $78(64.5)$ & $50(64.1)$ & $28(35.9)$ & $1.0(\mathrm{NS})$ \\
\hline
\end{tabular}

$\mathrm{OCP}=$ oral contraceptive pill.

HSE $=$ history of similar episodes

SAMPLE COLLECTION AND PROCESSING

Conventional HVS and rectal swabs were collected and plated onto Sabouraud agar, before incubation at $37^{\circ} \mathrm{C}$ for 48 hours. Vaginal washings were collected by introducing $10 \mathrm{ml}$ of sterile water into the vagina using a quill attached to a syringe. The washings were then re-aspirated into the syringe and transferred to a sterile Universal container. Each sample was serially diluted so that the number of viable colonies could subsequently be quantified. The neat washings and each dilution $(0.1 \mathrm{ml}$ volumes) were plated onto Sabouraud agar and incubated at $37^{\circ} \mathrm{C}$ for 48 hours. The number of colonies that grew was recorded and the results expressed as colony forming units (CFU) per $\mathrm{ml}$ of wash fluid.

Rectal and vaginal isolates were identified as Candida albicans on the basis of the germ tube test ${ }^{15}$ and the production of chlamydospores on Czapek Dox medium. Germ tube negative isolates were further identified using Auxocolor kits (Sanofi Pasteur, France). Five yeast colonies from each positive vaginal or rectal specimen were preserved using the Protect system (Technical Services Ltd, Heywood, UK) and kept at $-70^{\circ} \mathrm{C}$.

TYPING OF CANDIDA ISOLATES

DNA extraction

The method was adapted from Scherer and Stevens. ${ }^{16}$ Five yeast colonies from the vaginal washings and five yeast colonies from the rectal swab taken at the initial visit were inoculated onto separate Sabouraud agar plates and incubated for 48 hours. Five yeast colonies from vaginal washings were similarly cultured from each subsequent visit, if positive cultures were obtained. DNA was then extracted from each of these isolates.

Digestion and electrophoresis of DNA

The extracted DNA was digested using a specific restriction enzyme (EcoRI). The samples were then subjected to gel electrophoresis to separate the fragments into specific bands according to size.

\section{Southern blotting and hybridisation}

The electrophoresed DNA was blotted from the gel onto a nylon membrane, according to the method of Southern ${ }^{16}$ and identified by hybridisation with a $\mathrm{Ca} 3$ probe. ${ }^{17}$

ANTIFUNGAL SUSCEPTIBILITY TESTING

Antifungal susceptibility testing was performed by the broth microdilution method according to the National Committee for Clinical Laboratory Standards M27-A method. ${ }^{18}$ The antifungal agents used were clotrimazole (CTZ; Sigma) and fluconazole (FCZ; kindly provided by Pfizer, UK). The antifungal agents were tested at concentrations ranging from 64$0.125 \mathrm{mg} / \mathrm{l}$. The minimum inhibitory concentration (MIC) was assessed visually and defined as the concentration of antifungal that resulted in an $80 \%$ inhibition of growth of the test strain. For fluconazole, isolates with MICs less than or equal to $8 \mathrm{mg} / \mathrm{l}$ were defined as "sensitive," 16-32 mg/l as "susceptible dependent on dose," and $64 \mathrm{mg} / 1$ or greater as "resistant." For clotrimazole, isolates with MICs less than or equal to $1 \mathrm{mg} / 1$ were defined as sensitive, $2-8 \mathrm{mg} / 1$ as susceptible dependent on dose, and $16 \mathrm{mg} / \mathrm{l}$ or greater as resistant.

\section{DATA ANALYSIS}

The questionnaires were analysed using sPSS for Windows. Associations of Candida culture results with signs and symptoms were identified by bivariate analysis using the $\chi^{2}$ test, Pearson coefficient, and the $\mathrm{p}$ value calculated. Gels were analysed using the method of Schmid et $a l^{19}$ for DNA fingerprinting, calculation of similarity coefficient $\left(\mathrm{S}_{\mathrm{AB}}\right)$, and histogram and dendrogram genesis. $\mathrm{Ca} 3$ hybridisation patterns of five colonies per isolate were analysed for band position only in the size range of $2.25-19 \mathrm{~kb}$ and the results stored in the Dendron database. This constructs histograms and dendrograms by the unweighted pair group method. $S_{A B} S$ were recorded for each pair of strains from the linear list of the matrix coefficient, rather than branching points, as it is more accurate. In this study, the cut-off points for strain relatedness were lowered compared to other studies using this probe, as the band intensity was not included in the analysis, and because Dendron analysis only involved bands lower than $7.9 \mathrm{~kb}$. Genetically identical isolates were defined to have $S_{A B} s>0.96$, genetically similar but not identical isolates had $\mathrm{S}_{\mathrm{AB}} \mathrm{s}$ in the range $0.96-0.85$, and genetically unrelated isolates had $\mathrm{S}_{\mathrm{AB}} \mathrm{s}<0.85$.

\section{Results}

STUDY POPULATION

A total of 121 women were recruited to the study. The women had a mean age of 26 years (range 19-53 years). All had symptoms and signs suggestive of VVC, but only 78 of them $(65 \%)$ had positive Candida cultures at the initial visit. Table 1 shows the relation between symptoms and culture results in the study population at the initial visit.

MICROBIOLOGY RESULTS AT THE INITIAL VISIT Of the 78 women who had culture positive vaginal washings, $C$ albicans was isolated from 75, $C$ glabrata from two, and $C$ tropicalis from one. Rectal swabs were positive for Candida in 80 women, of whom 67 had concomitant initial positive vaginal washings. Again, $C$ albicans was the commonest species isolated from rectal swabs $(\mathrm{n}=78)$, followed by $C$ glabrata $(\mathrm{n}=1)$ and Saccharomyces cerevisiae $(\mathrm{n}=1)$. The patient with $C$ glabrata isolated from the rectal swab 


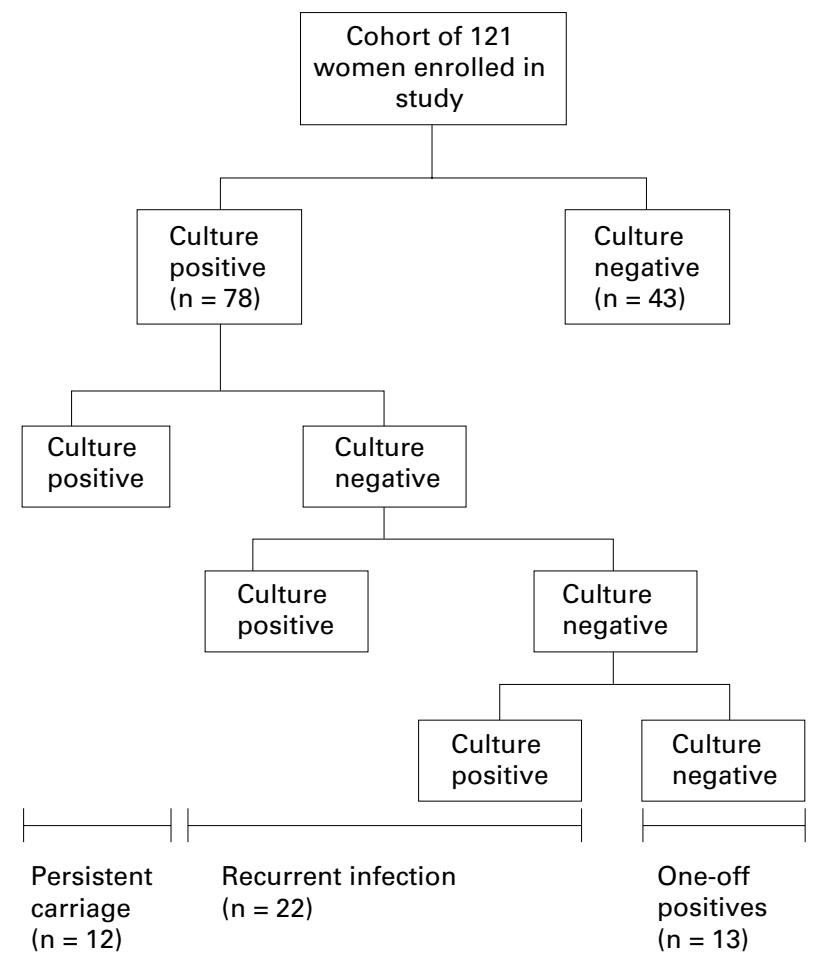

Figure 1 Classification of women in the study according to Candida culture results.

Table 2 Mean $\log _{10}$ viable count of Candida in women with persistent symptomatic or asymptomatic infection

\begin{tabular}{llll}
\hline & \multicolumn{2}{l}{$\begin{array}{l}\text { Mean } \log _{10} \text { viable Candida count } \\
\text { (95\% confidence limits) }\end{array}$} & \\
\cline { 2 - 3 } & Visit 1 & Visit 2 & P Value \\
\hline $\begin{array}{l}\text { Persistent carriage }(\mathrm{n}=12) \\
\text { Asymptomatic }(\mathrm{n}=9)\end{array}$ & $4.38(0.59)$ & $2.18(0.98)$ & $<0.05$ \\
Symptomatic $(\mathrm{n}=3)$ & $4.31(1.94)$ & $3.06(0.44)$ & NS \\
\hline
\end{tabular}

Data analysed by ANOVA.

also had $C$ glabrata in the vaginal washings. One patient with vaginal $C$ glabrata and one with vaginal $C$ tropicalis both had negative rectal cultures. The woman with the $S$ cerevisiae in the rectal swab had a negative vaginal culture.

The results for the HVS were broadly similar, with 72 of the 78 women with positive washings also having a positive HVS. In most cases, the results from the washings and HVS from the same woman were identical, but there were five women with positive washings and negative HVS and one woman with a positive HVS and negative washings.

CLINICAL OUTCOME

Of the 78 women who had positive vaginal washings at the initial visit, a total of 47 women completed the clinical study (either returned for four scheduled visits or experienced clinical recurrence) within the 3 month study period. These 47 women were classified into three groups according to their clinical outcome (fig 1):

- Thirteen women had an initial positive vaginal washing, but became and remained culture negative after therapy (one-off positives)

- Twelve women were culture positive at visit 2 despite therapy (persistent carriage)
- Twenty two women who were initially culture positive, became culture negative after therapy but again became culture positive, within the 12 week period (recurrent infection).

Of the 12 women with persistent carriage at 1 week post-treatment, nine were asymptomatic. Six of these nine women had a negative HVS, but positive vaginal washings on culture. Seven of the nine women continued to attend for follow up and seven subsequently developed symptoms requiring treatment.

VIABLE CANDIDA COUNTS AND RESPONSE TO TREATMENT

Viable Candida counts in vaginal washings taken at the initial visit were not predictive of clinical outcome. However, women with asymptomatic persistent infection had significantly lower viable Candida counts at visit 2 compared with visit $1(\mathrm{p}<0.05)$. In women with symptomatic persistent infection there was no significant difference between the counts at visits 1 and 2 (see table 2).

GENETIC RELATEDNESS BETWEEN VAGINAL AND RECTAL ISOLATES (PRETREATMENT)

Of the 66 women with positive cultures for $C$ albicans in both vagina and rectum, 52 had genetically identical isolates. In eight women the isolates were highly similar and in six they were unrelated.

GENETIC RELATEDNESS OF VAGINAL ISOLATES PRETREATMENT AND POST-TREATMENT

In women with recurrent infection, $\mathrm{S}_{\mathrm{AB}}$ scores between primary and subsequent vaginal isolates ranged from 1.00 to 0.52 . In 17 of the 22 women in the recurrent group, strain maintenance was seen, with 14 women having identical strains and three having highly similar strains. In the five remaining women, four had strain replacement and in one woman the Candida species changed from $C$ albicans to $C$ glabrata.

In the persistent carriage group, nine women had strain maintenance, two women had strain replacement, and in one patient the species of Candida isolated changed from $C$ albicans to $C$ parapsilosis. Figure 2 shows the Ca3 Southern blot hybridisation patterns of the Candida isolates from a patient who was culture positive at the initial visit as well as on the two subsequent visits (persistent carriage group). The blot demonstrates that strain maintenance was seen in this patient. Figure 3 shows the hybridisation patterns of isolates from two patients with recurrent infection; one illustrates strain maintenance and the other strain replacement.

ANTIFUNGAL SUSCEPTIBILITY TESTING

None of the isolates from the women in this study was resistant to fluconazole or clotrimazole (MIC ranges: $0.125-32 \mathrm{mg} / 1$ and $0.125-2$ $\mathrm{mg} / \mathrm{l}$ respectively). One post-treatment $C$ albicans isolate from a woman in the persistent group, was susceptible dependent on dose to fluconazole. Eight $C$ albicans isolates also 
$\begin{array}{lllllllllllllllllllll}\mathrm{C} & 1 & 2 & 3 & 4 & 5 & 6 & 7 & 8 & 9 & 10 & 11 & 12 & 13 & 14 & 15 & 16 & 17 & 18 & 19 & 20\end{array}$

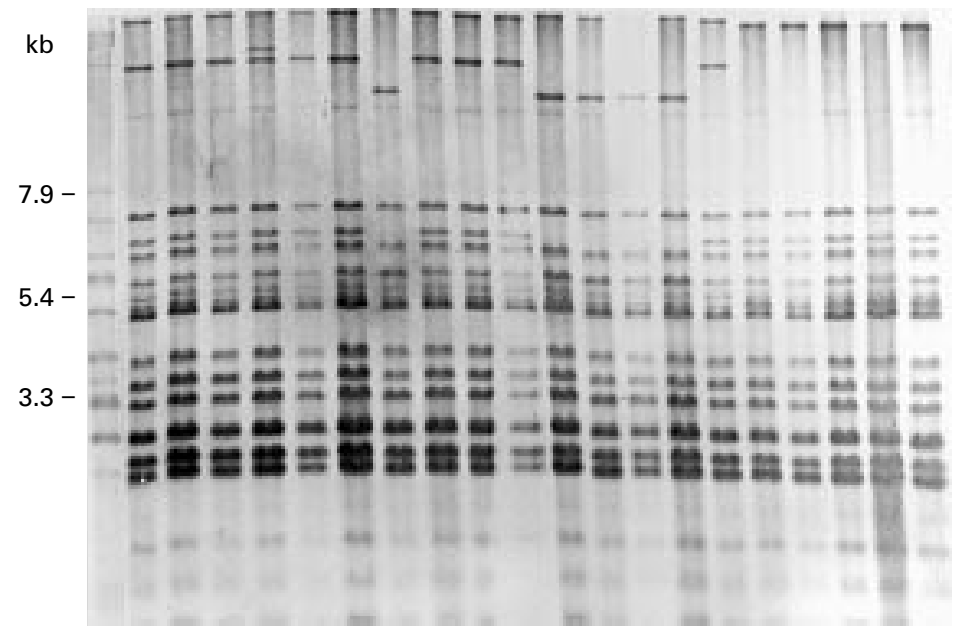

Figure 2 Ca3 hybridisation patterns of isolates from a woman in the persistent carriage group. Lanes 1-5 represent vaginal isolates taken at visit 1 and lanes 6-10 represent rectal isolates from visit 1. Lanes 11-15 represent vaginal isolates obtained at visit 2 when the patient was asymptomatic. Ten weeks after her initial visit, the patient again developed symptoms. Lanes 16-20 represent vaginal isolates obtained at this time. Strain maintenance occurred throughout with only minor variations in band pattern.

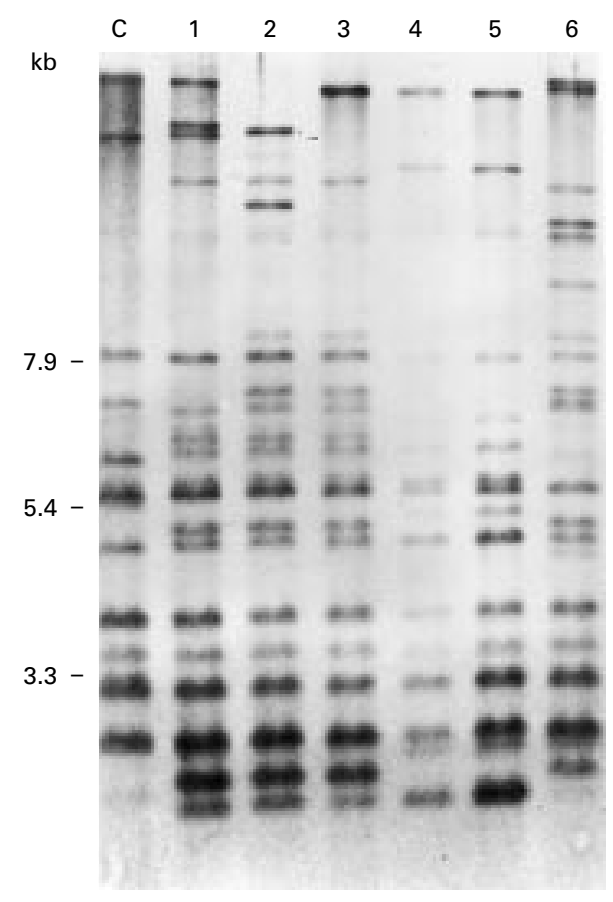

Figure 3 Southern blot hybridisation pattern of isolates from two women in the recurrent positive group. Lane $C$ is a reference Candida albicans isolate. Lanes 1-3 illustrate strain maintenance in one patient. Lanes 1 and 2 represent the vaginal and rectal isolates respectively, obtained at the initial visit. Lane 3 represents the vaginal isolate obtained during the recurrent episode. Lanes 4-6 illustrate strain replacement in another woman. Lanes 4 and 5 represent vaginal and rectal isolates obtained initially. Lane 6 represents the vaginal isolate obtained during the recurrent episode.

showed susceptibility dependent on dose to clotrimazole, three of which came from the same patient in the persistent carriage group.

\section{Discussion}

In this study, 121 women presented with symptoms suggesting acute vulvo-vaginal candidosis (VVC), yet only two thirds of them were culture positive for Candida species. Vulval itching and soreness were the only symptoms predictive of a culture positive vaginal washing, but overall, symptoms were non-specific, confirming the findings of previous published studies that diagnosis on the basis of history alone is unreliable..$^{20}$

Hopwood et $a l^{11}$ found a positive relation between viable yeast counts and symptoms and signs of VVC. We wondered whether viable Candida counts in vaginal washings taken at the initial visit would be predictive of the clinical outcome, but our results showed no association. However, in women with persistent infection after initial treatment, those who had higher viable Candida counts were more likely to be symptomatic.

$C$ albicans was by far the commonest isolate from both vagina and rectum $(96 \%$ of vaginal isolates and $98 \%$ of rectal isolates). These results are consistent with our previous observation $^{22}$ that there has been no increase in the incidence of non-albicans strains among women attending the genitourinary medicine clinic in Leeds since the introduction of "over the counter" antifungals.

Work in vitro has shown that the sensitivity of swabs as a sampling method for yeasts is low. ${ }^{3}$ In the laboratory, swabs will only yield positive cultures if they have been placed in a suspension containing at least $10^{3}$ viable organisms per ml. This study highlighted the discrepancy between culture results from HVS and vaginal washings. Of the 12 women with persistent carriage of yeast 1 week after treatment, half were culture negative by HVS, but positive by vaginal washings. Nine of the 12 women with persistent carriage in this study were asymptomatic; seven of them continued to attend and subsequently developed symptoms requiring re-treatment. Thus, in clinical practice some women treated for "recurrent infection" on the basis of HVS results may in fact have incomplete eradication of yeasts after treatment and should therefore be classed as persistent carriers.

The analysis of the genetic relatedness of vaginal isolates pretreatment and posttreatment demonstrated genotypic strain maintenance in the majority of women $(77 \%$ of women in the recurrent group and $75 \%$ of those in the persistent carriage group). There have been a number of other studies that have confirmed the genetic similarity of Candida species isolated during recurrent episodes of VVC (RVVC). ${ }^{13} 1423$ However, these studies only examined isolates obtained during symptomatic recurrence. Only a few studies have included specimens taken between symptomatic episodes, and have included very small numbers of patients. For example, Stein et $a l^{4}$ studied four women with RVVC, taking samples 1,7 , and 30 days after the treatment. Three had symptomatic recurrences with the same strain of $C$ albicans as was initially isolated from the vagina. Soll et $a l^{25}$ took repeated samples from various body sites in a single patient and found that strain maintenance occurred during three episodes of vaginal infection. A study examining chemoprophylaxis in women 
with RVVC included a larger number of women and vaginal samples were also taken between symptomatic recurrences. ${ }^{4}$ In this study, nearly half the women had strain replacement or a change of Candida species at the first symptomatic recurrence. However, as all the patients were receiving ketoconazole prophylaxis, this may have exerted a selective pressure encouraging the emergence of different strains or species of Candida.

In summary, our findings support the "vaginal relapse" hypothesis in women with RVVC and have important implications for the treatment of women with recurrent symptoms. We chose a single clotrimazole pessary (500 $\mathrm{mg}$ ) as treatment because this is the cheapest antifungal treatment available over the counter and is also frequently prescribed by general practitioners. Despite widespread usage of antifungal drugs, we and others have found no evidence of resistance to either fluconazole or clotrimazole in immunocompetent patients with recurrent infection or persistent carriage of Candida. ${ }^{22}{ }^{26} 27$ As antifungal resistance does not seem to be a problem in the general population, it may be appropriate to use more prolonged courses of antifungal treatment in women with RVVC in order to increase the chances of complete eradication of yeasts. One possible approach is an extended course of oral antifungals initially followed by regular prophylaxis, say once or twice monthly, with either vaginal pessaries or systemic therapy.

Funding for this project is gratefully acknowledged from the Special Trustees Research Scheme, Leeds Teaching Hospitals Special
Trust.

Contributors: SSE-D carried out the practical work associated Contributors: SSE-D carried out the practical work associated
with the project and contributed to the manuscript; MTR was with the project and contributed to the manuscript; MTR was
responsible for recruitment and examination of patients, clinical responsible for recruitment and examination of patients, clinical advice, and preparation of manuscript; HRA was responsible for
day to day supervision of the work, involved with antifungal day to day supervision of the work, involved with antifungal testing, and preparation of the manuscript; RCB provided
detailed advice on molecular methods and interpretation of results and contributed to the manuscript; EGVE was responsible for devising project and overall management, advice, interpretation of results, and contributed to the manuscript.

1 Fidel PL, Sobel JD. Immunopathogenesis of recurrent vulvovaginal candidiasis. Clin Microbiol Rev 1996;9:33548.

2 Sobel JD. Vaginitis and vaginal flora:controversies abound. Curr Opin Infect Dis 1996;9:42-7.

3 Odds FC. Genital candidosis. Clin Exp Derm 1982;7:345-

4 O'Connor MI, Sobel JD. Epidemiology of recurrent vulvovaginal candidiasis: identification and strain differen-

5 Meinhof WL. Demonstration of typical features of individual Candida albicans strains as a means of studying vidual Candida albicans strains as a means of studying
sources of infection. Chemotherapy 1982;28(Suppl 1):51-5.
6 Milne JD, Warnock DW. Effect of simultaneous oral and vaginal treatment on the rate of cure and relapse in vaginal candidosis. Br F Vener Dis 1979;55:362-5.

7 Vellupillai S, Thin RN. Treatment of vulvovaginal yeast infection with nystatin. Practitioner 1977;219:897-901.

8 Nystatin Multicentre Study Group. Therapy of candidal vaginitis:the effect of eliminating intestinal Candida. $A m \mathcal{F}$ Obstet Gynecol 1986;155:651-5.

9 Spinillo A, Carratta L, Pizzoli G, et al. Recurrent vaginal candidiasis. Results of a cohort study of sexual transmission and intestinal reservoir. F Reprod Med 1992;37:343-7.

10 Bisschop MPJM, Merkus JMWM, Scheygrond H, et al. Co treatment of the male partner in vaginal candidosis: a double blind randomised control study. Br F Obstet Gynaecol 1986;93:79-81.

11 Buch A, Christensen ES. Treatment of vaginal candidosis with natamycin and effect of treating the partner at the same time. Acta Obstet Gynecol Scand 1982;61:393-6.

12 Calderon-Marquez JJ. Itraconazole in the treatment of vaginal candidosis and the effect of treatment on the sexual partner. Rev Infect Dis 1987;9(Suppl 1):143-5.

13 Lockhart SR, Reed BD, Piersn CL, et al. Most frequent scenario for recurrent Candida vaginitis is strain maintenance with "substrain shuffling": demonstration by sequential DNA fingerprinting with probes $\mathrm{Ca} 3, \mathrm{C} 1$ and CARE2. $\mathcal{f}$ Clin Microbiol 1996;34:767-77.

14 Vazquez JA, Sobel JD, Demitriou R, et al. Karyotyping of Candida albicans isolates obtained longitudinally in women with recurrent vulvovaginal candidiasis. F Infect Dis 1994;170:1566-9.

15 Taschdjian CL, Burchall JJ, Kozinn PJ. Rapid identification of Candida albicans by filamentation on serum substitutes. Am F Dis Child 1960;99:212-15.

16 Southern EM. Detection of specific sequences of DNA fragments separated by gel electrophoresis. $f \mathrm{Mol} \mathrm{Biol}$ 1975;98:503-17.

17 Scherer S, Stevens DA. Application of DNA typing methods to epidemiology and taxonomy of Candida species. $\mathcal{F}$ Clin Micro 1987;25:675-9.

18 National Committee for Clinical Laboratory Standards. Reference method for broth dilution antifungal susceptibility testing of yeasts; approved standard. Wayne, Pennsylvania: National Committee for Clinical Laboratory Standards, 1997.

19 Schmid J, Voss E, Soll DR. Computer-assisted methods for assessing strain relatedness in Candida albicans by fingerprinting with the moderately repetitive sequence Ca3. F Clin Microbiol 1990;28:1236-43.

20 Kinghorn GR. Vulvovaginal candidosis. F Antimicrobiol Chemother 1991;28(Supp A):59-66.

21 Hopwood V, Crowley T, Horrocks CT, et al. Vaginal candidosis: relation between yeast counts and symptoms and clinical signs in non-pregnant women. Genitourin Med 1988;64:331-4.

22 Walker PP, Reynolds MT, Ashbee HR, et al. Vaginal yeasts in the era of "over the counter" antifungals. Sex Transm Inf 2000;76:437-8.

23 Mercure S, Poirier S, Lemay G, et al. Application of biotyping and DNA typing of Candida albicans to the epidemiology of recurrent vulvovaginal candidiasis. F Infect Dis 1993; 168:502-7.

24 Stein GE, Sheridan VL, Magee BB, et al. Use of rDNA restriction fragment length polymorphisms to differentiate strains of Candida albicans in women with vulvovaginal candidiasis. Diagn Microbiol Infect Dis 1991;14:459-64.

25 Soll DR, Galask R, Isley S, et al. Switching of Candida albicans during successive episodes of recurrent vaginitis. $\mathcal{F}$ Clin Microbiol 1989;27:681-90.

26 Fong IW, Bannatyne RM, Wong P. Lack of in vitro resistance of Candida albicans to ketoconazole, itraconazole and clotrimazole in women treated for recurrent vaginal candidiasis. Genitourin Med 1993;69:44-6.

27 Lynch ME, Sobel JD, Fidel PL. Role of antifungal drug resistance in the pathogenesis of recurrent vulvovaginal candidiasis. F Med Vet Mycol 1996;34:337-9. 